\title{
Intensive Care Society of Ireland - Guidelines for management of the potential organ donor (2018-2nd edition)
}

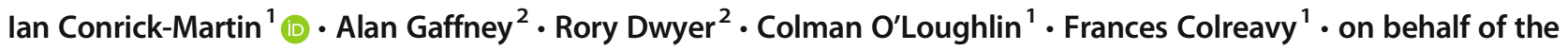 \\ Intensive Care Society of Ireland
}

Received: 5 February 2019 / Accepted: 7 February 2019 /Published online: 5 March 2019

(C) Royal Academy of Medicine in Ireland 2019

\section{Introduction}

Advances in transplant surgical techniques and immunosuppressive therapies have led to increasing numbers of patients with end-stage organ failure being treated with an organ transplant. There is increasing evidence that success rates of transplantation can be improved with optimal management of the potential organ donor [1]. Thus it is important, after diagnosis of brain death, that optimal physiological support is maintained to allow the option of organ donation if this was the patient's known wish.

The guidelines as outlined should be implemented only after the diagnosis of brain death in patients who are potential organ donors. Before the diagnosis of brain death, patients should be managed as appropriate for their underlying condition. After the diagnosis of brain death, should the patient have wished to become an organ donor, treatment becomes oriented toward optimising organs that may be retrieved for transplantation rather than toward neuroprotection.

Ian Conrick-Martin

iconrickmartin@mater.ie

Alan Gaffney

alangaffney@beaumont.ie

Rory Dwyer

rorydwyer@me.com

Colman O'Loughlin

coloughlin@mater.ie

Frances Colreavy

fcolreavy@mater.ie

1 Department of Critical Care Medicine, Mater Misericordiae University Hospital, Dublin 7, Ireland

2 Department of Anaesthesia and Intensive Care Medicine, Beaumont Hospital, Dublin 9, Ireland
Appropropriate adjustments must be made for paediatric organ donors; these guidelines are written solely for adults. The guidelines are informed by critical appraisal of the relevant literature, recommendations/guidelines from jurisdictions such as Canada [2], USA [3], UK [4] and Australian \& New Zealand [5], and our own experience of clinical practice in Ireland.

These guidelines are primarily aimed at supporting those Intensive Care Units (ICUs) where potential organ donors are managed infrequently. Where more advanced or specialist advice is needed, we suggest early involvement of an Organ Donor Nurse Manager (ODNM) or Clinical Lead in Organ Donation (CLOD).

\section{Haemodynamic monitoring and therapy}

The objectives of haemodynamic management are to optimise organ perfusion in the potential organ donor patient. The haemodynamic goals and targets below are recommended during the entire donor management phase of care.

\section{General haemodynamic goals}

- Maintain euvolaemia.

- Reduce vasoconstrictors as far as possible to maintain a normal afterload.

- Optimise cardiac output ideally without, or if required with, minimum doses of $\beta$-agonists or other inotropes.

\section{Bedside target}

- Blood pressure: Mean arterial pressure $60-70 \mathrm{mmHg}$

- Central venous pressure: $6-10 \mathrm{mmHg}$

- Urine output: $1-3 \mathrm{ml} / \mathrm{kg} / \mathrm{h}$ 


\section{Echocardiography}

- Left ventricular ejection fraction: at least $45 \%$

\section{Pulmonary artery catheter (if present)}

- Cardiac index: $>2.41 / \mathrm{min} / \mathrm{m}^{2}$

- Pulmonary capillary wedge pressure: $8-12 \mathrm{mmHg}$

It is important to note that these variables are goals of therapy; successful organ retrieval and transplantation is still possible with haemodynamic variables outside these targets.

\section{Volume status and fluid therapy}

Hypovolaemia is common following brain death due to, e.g. mannitol-induced osmotic diuresis and/or polyuria secondary to diabetes insipidus. Preferred fluid choices are the isotonic crystalloids; Ringers lactate (Hartmann's solution) or $0.9 \%$ saline. Use of the latter may be limited by hyperchloraemic acidosis and/or management of dysnatraemia. Colloid solutions as a bolus infusion have a role in acute intravascular volume expansion. Red cell transfusion may be used to target haemoglobin above $7 \mathrm{~g} / \mathrm{dl}$, which in the absence of evidence is the recommended haemoglobin threshold (for non-organ donor) ICU patients.

A central venous catheter is routinely required; ideally in the internal jugular or subclavian veins. Maintaining a CVPtargeted approach of $<10 \mathrm{mmHg}$, albeit with combined hormonal therapy, has been shown to significantly improve rates of heart and lung procurement without affecting the availability of kidneys for transplantation [6].

Dynamic indicators, e.g. response of CVP to fluid challenge, passive leg raising or pulse pressure variability, are accepted as a better guide to fluid therapy than static measurements such as a single CVP measurement. Semi-invasive haemodynamic monitoring tools using pulse contour analysis to assess stroke volume variation may also be useful.

\section{Cardiovascular assessment and vasoactive medications}

The defining moment of brain death is associated with a catecholamine surge, often leading to tachycardia and extreme hypertension. There follows a systemic inflammatory response and marked vasodilation from loss of peripheral vasomotor tone. The effect of these haemodynamic changes on the myocardium is often visible on an ECG or echocardiogram (as regional wall motion abnormalities) in up to $42 \%$ of potential heart donors [7].

Initial echocardiographic findings of impaired right or left heart function may only be reflective of the brain death pathophysiological process and are therefore potentially reversible. Identification of a left ventricular ejection fraction <
$45 \%$ should prompt consideration of serial cardiac output monitoring to guide management.

Where practicable, a transthoracic echocardiogram (TTE) should be considered with all potential organ donors and, in particular, with potential heart donors, to better characterise baseline cardiac function as an aid to optimisation of organ perfusion. However, this should not delay referral of a potential organ donor to the National Organ Procurement Service for assessment regarding organ suitability.

When a donor heart is declined by the retrieval surgeons due to poor function, consideration should be given to allowing time for further optimisation (up to $72 \mathrm{~h}$ ) as ventricular function may improve and thus facilitate successful retrieval and transplantation [8].

If correction of a volume deficit fails to achieve the threshold haemodynamic goals, then use of vasoactive medications is required. The choice of vasoactive agent will be informed by knowledge of cardiac function and circulatory status.

\section{First-line vasoactive agent}

- Vasopressin $(0.5-2.4 \mathrm{IU} / \mathrm{h})$ is the recommended firstline agent for the vasodilatory shock state associated with brain death. This low dose of vasopressin aids restoration of vascular tone and treats diabetes insipidus via an antidiuretic effect in the distal nephron.

- Vasopressin has the potential to cause excessive vasoconstriction and regular observation of the peripheries for malperfusion is necessary. This is particularly the case in patients with impaired heart function.

\section{Second-line vasoactive agents}

- Noradrenaline or phenylephrine may be used as secondline agents particularly in vasodilatory shock. The dose of noradrenaline should be kept to the minimum required as its use has been associated with reduced right ventricular function and reduced 1-year survival in heart transplant recipients [9].

- Dobutamine or adrenaline may be used for inotropic support when primary ventricular dysfunction is identified.

\section{$\beta$-Agonist therapy caution}

Pure vasopressors, e.g. vasopressin and phenylephrine, should be distinguished from vasopressors with $\beta$ agonist activity (e.g. noradrenaline, adrenaline) and pure $\beta$-agonists (e.g. dobutamine and isoprenaline). $\beta$-agonist 
therapy has the potential to cause adverse effects on heart donors secondary to increasing myocardial oxygen demand, depletion of myocardial adenosine triphosphate (ATP) and downregulation of $\beta$-receptors [10]. The Canadian \& US guidelines stipulate that the dose of dobutamine should be kept $<10 \mu \mathrm{g} / \mathrm{kg} / \mathrm{min}$.

If arterial hypertension occurs, catecholamines should be weaned before decreasing vasopressor infusion rates.

3. Haemodynamically unstable donors unresponsive to fluid therapy and vasoactive medications

Combined hormonal therapy includes the administration of thyroid hormone and glucocorticoids (see below). It is frequently given on initiation of donor management or may be reserved for patients with haemodynamic instability or impaired ventricular function on echocardiography. High-dose methylprednisolone diminishes the inflammatory vasodilatory response following brain death. There is some poor-quality evidence of the beneficial effects of hormonal therapy on improving haemodynamic function and increasing rate of heart transplantation [11-13].

\section{Respiratory management}

Pulmonary dysfunction is common in the potential organ donor due to pneumonia, aspiration of gastric contents, neurogenic pulmonary oedema, pulmonary trauma or ARDS (which itself may be secondary to acquired brain injury). This has led to a lower conversion rate of donor lungs for transplantation than that of other organs.

\section{General principles}

The utilisation of lung-protective ventilation, as described below, has been shown to have a significant impact on improving conversion rates of donor lungs and its use is strongly recommended.

It is also strongly recommended that during brain stem testing, the apnoea test be performed in the presence of positive end-expiratory pressure (PEEP), e.g. with a partially closed valve on a C-Circuit or with the use of an in-line PEEP valve, rather than with an open circuit, e.g. $\mathrm{O}_{2}$ insufflation via a suction catheter. The latter may cause clinically significant derecruitment of lung units leading to sub-optimal oxygenation; these can be very challenging to re-recruit.

\section{Standard ventilation}

- Routine physiotherapy, tracheal suctioning and oral care should be no different from usual ICU practice. Strict asepsis should be continued during tracheal toilet.
- A 30-degree head-up position should be adopted unless otherwise contraindicated.

- Endotracheal/tracheostomy cuff pressure should be kept $\leq 25 \mathrm{cmH}_{2} \mathrm{O}$.

\section{Ventilatory guidelines and optimisation of oxygenation}

- Tidal volumes of $6-8 \mathrm{ml} / \mathrm{kg}$ ideal body weight are recommended.

- Plateau inspiratory pressures $<30 \mathrm{cmH}_{2} \mathrm{O}$.

- PEEP of 5-10 $\mathrm{cmH}_{2} \mathrm{O}$ (or higher if clinically indicated) is recommended as a routine.

- Lung recruitment may be achieved by cautious periodic increases in PEEP up to $15 \mathrm{cmH}_{2} \mathrm{O}$ or by short sustained inflations (inspiratory pressures of $<30 \mathrm{cmH}_{2} \mathrm{O}$ ). This should be performed with great care given the potential for haemodynamic instability, particularly in this patient cohort.

- Diuresis to euvolaemia should be initiated if fluid overload occurs.

A lung-protective ventilatory strategy (minimising ventilatorinduced lung injury in patients with ARDS) is defined by plateau inspiratory pressures $<30 \mathrm{cmH}_{2} \mathrm{O}$, appropriate application of PEEP and tidal volumes of $6-8 \mathrm{ml} / \mathrm{kg}$ ideal body weight. This strategy should be used in the setting of the potential organ donor as it has been shown to correlate with increased conversion rates of donor lungs for transplantation $[14,15]$.

\section{Ventilatory targets}

- $\mathrm{PaCO}_{2}$ : Normocapnoea 4.8-5.8 $\mathrm{kPa}(36-44 \mathrm{mmHg})$ titrated to $\mathrm{pH} 7.35-7.45$

- FiO2: Lowest FiO2 to maintain $\mathrm{PaO}_{2} \geq 10 \mathrm{kPa}$ $(80 \mathrm{mmHg})$

\section{Role of bronchoscopy}

- Brochoscopic suctioning may be required if there are concerns regarding mucous plugging or particulate matter causing mechanical obstruction \pm distal collapse. Bronchoscopic sampling/lavage should be sent for culture and sensitivity. Lavage volumes should be minimised to reduce extra-vascular lung water.

\section{Role of steroids}

- Glucocorticoid administration (see the "Combined Hormonal Therapy" section) may reduce the inflammatory response and degree of acute lung injury [16]. 


\section{Post organ retrieval optimisation}

- Consideration should be given to post-retrieval lung reconditioning with ex vivo lung perfusion techniques [17].

\section{Diabetes insipidus and hypernatraemia}

With regard to serum sodium concentration, the maximum threshold for clinical brainstem testing is $155 \mathrm{mmol} / \mathrm{L}$. However, the target serum sodium for managing the potential organ donor should be the normal range (135-145 mmol/L) [18]. Similarly, normal values of potassium, magnesium, phosphate and calcium should be targeted. Urine output should be kept between 1 and $3 \mathrm{ml} / \mathrm{kg} / \mathrm{h}$.

Central diabetes insipidus (DI) is common following severe brain injury and brain death. A decrease in antidiuretic hormone $(\mathrm{ADH})$ release causes the kidneys to produce large volumes of dilute urine. This loss of water results in hyperosmolar hypernatraemia. Hypernatraemia may be associated with hepatic dysfunction and graft loss [19]. Large intravascular volume loss may lead to decreased organ perfusion and ultimately, organ failure. It is important to have a high degree of suspicion for the development of DI and to diagnose and treat it promptly when it does occur.

DI should be suspected when there is a significant increase in urine output associated with a rising serum sodium concentration. Blood should be sent for serum sodium concentration and osmolarity and urine for urine osmolarity and specific gravity. Any delay in management should be avoided while waiting for the results of these confirmatory tests.

\section{Features of diabetes insipidus}

1. High urine output $>4 \mathrm{ml} / \mathrm{kg} / \mathrm{h}$ (polyuria due to low $\mathrm{ADH}$ levels)

2. Increasing serum sodium $>145 \mathrm{mmol} / \mathrm{L}$ (hypernatraemia due to loss of serum water)

3. Increasing serum osmolarity $>300 \mathrm{mOsm} / 1$ (concentrated serum due to loss of water)

4. Decreasing urine osmolarity $<300 \mathrm{mOsm} / 1$ (dilute urine due to excess water)

5. Decreasing urine specific gravity $<1.005$ (dilute urine due to excess water)
Management of diabetes insipidus

(1) Replace ongoing fluid losses and any existing fluid deficit with hypotonic fluid.

- Start with $0.45 \%$ saline replacing the previous hour's urine output $\mathrm{ml}$ for $\mathrm{ml}$ in addition to further volume replacement for any pre-existing intravascular volume deficit.

- If it is difficult to lower serum sodium concentration, despite adequate fluid replacement with $0.45 \%$ saline, it is reasonable to change to Solution 18 or $5 \%$ dextrose. However, these may lead to hyperglycaemia or very rapid falls in serum sodium concentration particularly when combined with ADH replacement therapy such as vasopressin and/or 1-desamino-D-arginine vasopressin (DDAVP).

- Enteral $\mathrm{H}_{2} \mathrm{O}$ administration may also be useful

(2) If urine output is $>3 \mathrm{ml} / \mathrm{kg} / \mathrm{h}$, add an infusion of intravenous vasopressin and/or intermittent subcutaneous or intravenous DDAVP.

- If vasopressor support is required, IV vasopressin should be infused at a rate of $0.5-2.4 \mathrm{IU} / \mathrm{h}$. However, commonly this alone is not adequate to control diuresis and DDAVP should be given as soon as DI is suspected (see below).

- DDAVP is an analog of arginine vasopressin with enhanced antidiuretic potency, negligible vasopressor activity and a prolonged half-life compared to vasopressin. The dose of DDAVP in adults is $1-2 \mu \mathrm{g}$ subcutaneously or intravenously followed by 1-2 $\mu \mathrm{g}$ subcutaneously or intravenously as required to achieve a urine output $<3 \mathrm{ml} /$ $\mathrm{kg} / \mathrm{h}$.

The management of DI requires frequent assessment of urine output and serum sodium concentration until both return to the normal range.

\section{Glycaemic control and nutrition}

Hyperglycaemia is common in potential organ donors due to administration of dextrose-based solutions, peripheral insulin resistance, catecholamine administration and use of glucocorticoids. The major consequences of hyperglycaemia include osmotic diuresis, ketosis and potential pancreatic graft dysfunction following transplantation. Hyperglycaemia should be managed as per usual ICU practice. 
- Aim for a blood glucose level $\leq 10 \mathrm{mmol} / \mathrm{L}$ with an IV insulin infusion as needed.

- Routine enteral nutrition should be initiated or continued as tolerated.

- Parenteral nutrition should not be initiated; however, when it has been initiated it should be continued.

- Avoid routine use of dextrose-based solutions.

\section{Thermoregulation}

Hypothermia is very common in the potential organ donor due to loss of hypothalamic thermoregulatory control mechanisms, exposure to cold ambient temperatures or infusions of cold intravenous fluids/blood products. The potential adverse consequences of hypothermia include arrhythmias, myocardial depression, hypotension, hyperglycaemia and coagulopathy.

- Hypothermia should be anticipated and heat loss prevented; it is very often easier to prevent hypothermia than to correct it.

- Active heating measures are often needed including warming blankets, fluid warmers, heated humidifiers in ventilator circuits and increasing the ambient temperature.

- Aim for a core temperature $>36^{\circ} \mathrm{C}$ (i.e. normothermia).

\section{Antimicrobial therapy}

The principles of antimicrobial therapy are similar to those for ICU patients who are not potential organ donors.

- If infection in a potential organ donor is suspected, sampling of blood, urine, sputum, surgical wounds etc. (for culture and sensitivity) should be performed as is normal practice for any ICU patient.

- Advice should be sought from a Microbiology or Infectious Diseases Specialist to determine the significance of any positive culture results.

- Antimicrobial therapy should ideally be based on the results of gram stain or culture samples but may be empiric, based on likely pathogens causing infection.

- Nephrotoxic antimicrobials should be avoided if possible.

- The use of prophylactic antimicrobials is not indicated.
If there are clinical signs of sepsis, organ retrieval teams may choose to wait for the result of blood cultures to ensure the potential donor is not bacteraemic (which could adversely affect a potential transplant recipient). However, it is important to note that this is not an absolute contraindication to organ donation.

\section{Haematological management}

- Anaemia is common and may be exacerbated by haemodilution with clear fluid administration, bleeding, coagulopathy or a combination of these.

- The optimal haemoglobin concentration in the potential organ donor population is unknown. A target haemoglobin level of $9 \mathrm{~g} / \mathrm{dl}$ is appropriate to optimise cardiopulmonary function/oxygen delivery in the face of haemodynamic instability. A level of $7 \mathrm{~g} / \mathrm{dl}$ is the suggested lowest acceptable limit in stable patients.

- Drawing of blood for donor serology and tissue typing should ideally occur before transfusions are administered.

- There are no defined targets for platelet levels, INR, PT or APPT. Platelet, plasma or fibrinogen administration is indicated only with clinically significant bleeding or coagulopathy. Advice should be sought from a Coagulation or Haematology Specialist.

- CMV-negative blood products should be used.

- Thromboprophylaxis (mechanical and/or pharmacological) should, unless otherwise contraindicated, be used as per normal ICU practice.

\section{Combined hormonal therapy}

Hormonal therapy following brain death may include (1) vasopressin, (2) glucocorticoids, (3) thyroid hormone, and (4) insulin. These drugs are administered with the aim of optimising the numbers of organs retrieved and/or improving graft function following transplantation. There is a relatively low level of evidence that hormonal therapy achieves either of these aims. However, it is likely that their use is safe.

\section{Vasopressin}

A retrospective review suggested that the use of vasopres$\sin$ is associated with an increase in the numbers or organs retrieved for transplantation [20]. If a vasopressor is needed then vasopressin is recommended as the first-line agent, regardless of whether diabetes insipidus is present. 


\section{Glucocorticoids}

The evidence for the administration of glucocorticoids is low and is based mainly on the benefits shown in observational studies. Small, poor-quality randomised controlled trials showed no differences in outcomes for graft survival. However, there may be a role for their use in shock reversal in the haemodynamically unstable potential organ donor.

\section{Thyroid hormone}

The role of thyroid hormone replacement therapy is controversial. A 2012 systematic review and meta-analysis failed to show a role for routine administration of thyroid hormone [21]. However, a subsequent large-scale retrospective review showed a moderate increase in transplantable organs and improved graft function when thyroid replacement therapy was instituted [22].

Thyroid hormone replacement may have a role in the haemodynamically unstable organ donor patient.

\section{Insulin}

An intravenous insulin infusion should be administered as needed targetting a blood glucose level 5-10 $\mathrm{mmol} / \mathrm{L}$.

Given the low level of evidence for combined hormonal therapy, it is not necessary to automatically commence such therapies once brain death has occurred. However, the risks associated with use of these therapies are low [23]. Therefore, it is reasonable to consider instituting combined hormonal therapy, particularly where there is ongoing haemodynamic instability or where heart transplantation is being considered but cardiac function is poor (in order to limit the potential adverse effects of exogenous catecholamines).

\section{Drug doses for combined hormonal therapy}

- Vasopressin: 1 IU IV bolus followed by $0.5-2.4$ IU/h IV infusion

- Methylprednisolone: $1 \mathrm{~g} \mathrm{IV}$ every $24 \mathrm{~h}$

- T3 (tri-iodothyronine): $4 \mu \mathrm{g}$ IV bolus followed by IV infusion at $3 \mu \mathrm{g} / \mathrm{h}$

- Insulin: IV infusion as per local ICU protocol

\section{Timing and conduct of organ retrieval}

There is increasing recognition of the benefit of taking the necessary time in ICU to optimise potential donors' organ function in order to improve transplant recipient outcome. Targetted therapies can improve reversible organ dysfunction including myocardial stunning, impaired pulmonary gas exchange, sepsis and acute impairments in renal or hepatic function.

This treatment period may last up to $72 \mathrm{~h}$ and should be accompanied by frequent re-evaluation to demonstrate improvement in organ function toward defined targets. The benefits of allowing such periods of time need to be balanced against the risks of potential donor deterioration in the face of, e.g. cardiovascular instability and that of increasing emotional distress in the potential organ donor's family. Discussion between the treating intensive care team and organ retrieval teams can be helpful.

Final decisions regarding suitability for organ retrieval and transplantation rest with the relevant organ retrieval team.

The organ retrieval procedure itself can take up to $6 \mathrm{~h}$ (or longer) to complete; this is often dependent on multiple clinical and non-clinical factors. A volatile anaesthetic agent and muscle relaxant are normally administered to ensure optimal operating conditions (although 'anaesthesia' to prevent awareness is not required per se in a brain dead patient who is, by definition, deceased). Active donor management to optimise the condition of organs should continue until the organs are removed. At the end of the procedure, mechanical ventilation is discontinued and the endotracheal tube is removed. Patient dignity including care of the patient's body is of paramount importance throughout.

\section{Psychological issues for staff and relatives}

Staff caring for patients who are potential organ donors need to have a clear understanding of the moral, ethical, legal and clinical principles involved in caring for such patients who, after diagnosis of brain death, are deceased. Usual end-of-life care, including particular attention to patient dignity, should continue throughout this period. Ongoing education and training of medical and nursing staff is therefore vital; the Joint Faculty of Intensive Care Medicine of Ireland run an annual training course (Irish Donor Awareness Program) which may be helpful in this regard.

These cases can be psychologically demanding both in the clinical management of the individual patient and when dealing with relatives who can be understandably distressed at what is a traumatic time for them [24]. Junior medical and nursing staff in particular need support to optimally manage these scenarios. We suggest that, when appropriate, those with experience and training in these challenging areas (e.g. Organ Donor Nurse Managers or Clinical Leads in Organ Donation) be involved early, to facilitate open and clear lines of communication amongst all parties.

After an organ donation episode, a debrief can be helpful to support staff and/or donor families and should be facilitated where appropriate. 
Management of the Potential Organ Donor - Summary

Success rates of organ transplantation can be improved with optimal management of the potential organ donor.

Haemodynamics
- Maintain euvolemia
- CVP $60-70 \mathrm{mmHg}$
: 6 - $10 \mathrm{mmHz}$ output $1-3 \mathrm{ml} / \mathrm{kg} / \mathrm{hr}$
- C Ejection Fraction $>45 \%$

\section{Monitoring}

- Place an arterial line and a central venous catheter (jugular/subclavian veins)

Blood Pressure

- Reduce vasoconstrictors as far as possible Use vasopressin as first-line vasopressor (if required)

Volume Replacement

- Use isotonic crystalloids for volume replacement if required e.g Hartmann's solution

Cardiac Output

Optimise cardiac output with inotropes if required (when euvolaemic)

Echocardiography may be useful to guide fluid therapy/vasoactive medications

Myocardial Dysfunction

- Consideration should be given to allow up to 72 hours for potentially reversible myocardial dysfunction to improve
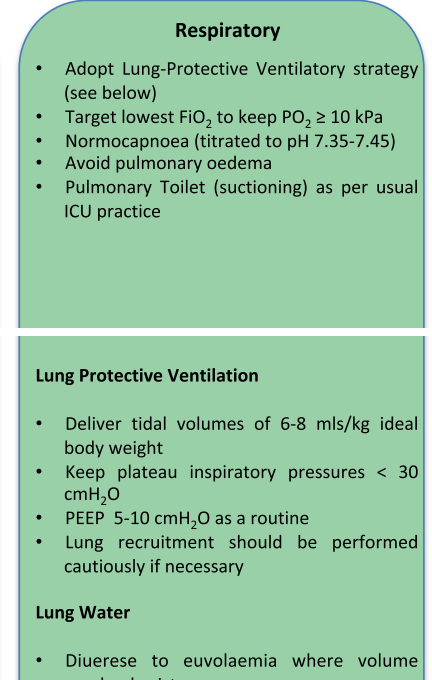

Diuerese to
overload exists

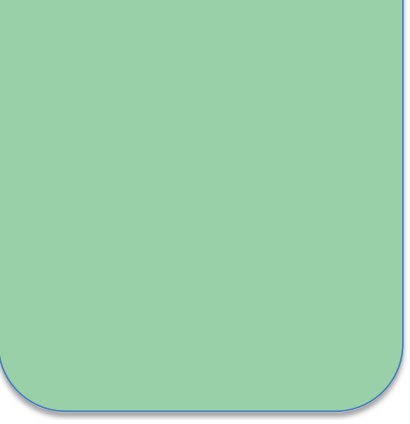

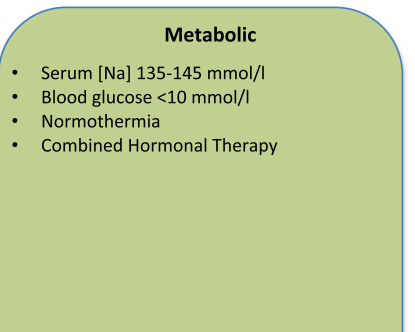

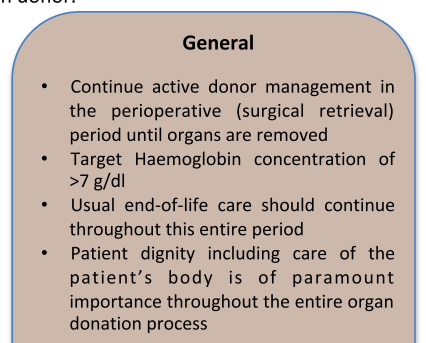

\section{Diabetes Insipidus}

- DI results in large volumes of dilute urine with rise in serum sodium

Anticipate

Treat with DDAVP +/-Vasopressin

- Replace volume deficit

Replace ongoing fluid losses with, in the first instance, $0.45 \%$ saline replacing the previous hour's urine output $\mathrm{ml}$ for $\mathrm{ml}$

Hyperglycaemia

- IV insulin infusion as needed

Hypothermia

- Anticipate

Prevent heat loss

Actively warm if necessary

Combined Hormone Therapy

Consider 1) Vasopressin, 2) Glucocorticoids, 3) Thyroid hormone, and 4) Insulin

Relatively low level of evidence that hormonal therapy improves the number of successfully retrieved organs or outcome following organ transplantation

However it is likely that use of hormonal

therapy is safe

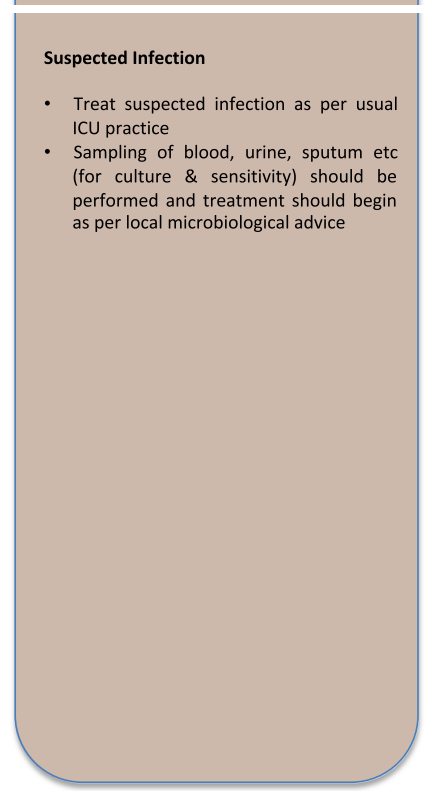

Acknowledgements Thanks to Colm Kavanagh, Printing Dept, Beaumont Hospital, for formatting this document.

\section{Compliance with ethical standards}

Competing interests The authors declare that they have no competing interests.

Publisher's note Springer Nature remains neutral with regard to jurisdictional claims in published maps and institutional affiliations.

\section{References}

1. Patel MS, De La Cruz S, Sally MB et al (2017) Active donor management during the hospital phase of care is associated with more organs transplanted per donor. J Am Coll Surg 225(4):525-531

2. Shemie SD, Ross H, Pagliarello J et al (2006) Organ donor management in Canada: recommendations of the forum on medical management to optimize donor organ potential. CMAJ 174(6): S13-S32

3. Kotloff RM, Blosser S, Fulda GJ, Malinoski D, Ahya VN, Angel L, Byrnes MC, DeVita M, Grissom TE, Halpern SD, Nakagawa TA, Stock PG, Sudan DL, Wood KE, Anillo SJ, Bleck TP, Eidbo EE, Fowler RA, Glazier AK, Gries C, Hasz R, Herr D, Khan A,
Landsberg D, Lebovitz DJ, Levine DJ, Mathur M, Naik P, Niemann CU, Nunley DR, O'Connor KJ, Pelletier SJ, Rahman O, Ranjan D, Salim A, Sawyer RG, Shafer T, Sonneti D, Spiro P, Valapour M, Vikraman-Sushama D, Whelan TP, Society of Critical Care Medicine/American College of Chest Physicians/ Association of Organ Procurement Organizations Donor Management Task Force (2015) Management of the Potential Organ Donor in the ICU: Society of Critical Care Medicine/American College of Chest Physicians/Association of Organ Procurement Organizations Consensus Statement. Crit Care Med 43:1291-1325

4. NHS Blood \& Transplant. Donor optimisation: Guidance around selecting potential DBD Donors. Available at https://www.odt.nhs.uk/ deceased-donation/best-practice-guidance/donor-optimisation/ (Accessed March 2018)

5. Australia and New Zealand Intensive Care Society (ANZICS): The ANZICS statement on death and organ donation 2013 (Edition 3.2). Available at http://www.anzics.com.au/Pages/DaOD.aspx (Accessed March 2018)

6. Abelnour T, Rieke S (2002) Relationship of hormonal resuscitation therapy and central venous pressure on increasing organs for transplant. J Heart Lung Transplant 28:480-485

7. Dujardin KS, McCully RB, Wijdicks EFM et al (2001) Myocardial dysfunction associated with brain death: clinical, echocardiographic, and pathological features. J Heart Lung Transplant 20:350-357

8. Dimarakis I, Banner NR, Rushton S, Wong HSE, Berman M, Howell N, Payne J, Dark J, Mehew J, Venkateswaran R (2018) 
The interval between brainstem death and cardiac assessment influences the retrieval of hearts for transplantation. Eur J Cardiothorac Surg 53:1135-1143

9. Stoica S, Satchithananda DK, White P et al (2004) Noradrenaline use in the human donor and relationship with load-independent ventricular contractility. Transplantation 78:1193-1197

10. D'Amico TA, Meyers CH, Koutlas TC, Peterseim DS, Sabiston DC Jr, van Trigt P, Schwinn DA (1995) Desensitization of myocardial betaadrenergic receptors and deterioration of left ventricular function after brain death. J Thorac Cardiovasc Surg 110(3):746-751

11. Jeevanandam V, Todd B, Regillo T et al (1994) Reversal of donor myocardial dysfunction by triiodothyronine replacement therapy. J Heart Lung Transplant 13(4):681-687

12. Wheeldon DR, Potter CDO, Oduro A et al (1995) Transforming the "unacceptable" donor: outcomes from adoption of a standardized donor management technique. J Heart Lung Transplant 14:734-742

13. Salim A, Vassiliu P, Velmahos GC, Sava J, Murray JA, Belzberg H, Asensio JA, Demetriades D (2001) The role of thyroid hormone Administration in Potential Organ Donors. Arch Surg 136:13771380

14. Mascia L, Pasero D, Slutsky AS, Arguis MJ, Berardino M, Grasso S, Munari M, Boifava S, Cornara G, Della Corte F, Vivaldi N, Malacarne P, del Gaudio P, Livigni S, Zavala E, Filippini C, Martin EL, Donadio PP, Mastromauro I, Ranieri VM (2010) Effect of a lung protective strategy for organ donors on eligibility and availability of lungs for transplantation: a randomized controlled trial. JAMA 304:2620-2627

15. Angel LF, Levine DJ, Restrepo MI, Johnson S, Sako E, Carpenter A, Calhoon J, Cornell JE, Adams SG, Chisholm GB, Nespral J, Roberson A, Levine SM (2006) Impact of a lung transplantation donor-management protocol on lung donation and recipient outcomes. Am J Respir Crit Care Med 174(6):710-716
16. Dupuis S, Amiel J-A, Desgroseilliers M, Williamson DR, Thiboutot Z, Serri K, Perreault MM, Marsolais P, Frenette AJ (2014) Corticosteroids in the management of brain-dead potential organ donors: a systematic review. Br J Anaesth 113(3):346-359

17. McKeown DW, Bonser RS, Kellum JA (2012) Management of the heartbeating brain-dead organ donor. Br J Anaesth 108(S1):i96i107

18. Dwyer R, Motherway C, Phelan D (on behalf of Intensive Care Society of Ireland). Diagnosis of Brain Death in adults; guidelines. Available at http://www.intensivecare.ie/publications/ (Accessed April 2018)

19. Mangus RS, Fridell JA, Vianna RM, Milgrom ML, Chestovich P, Vandenboom C, Tector AJ (2010) Severe hypernatremia in deceased iver donors does not impact early transplant outcome. Transplantation 90(4):438-443

20. Plurad DS, Bricker S, Neville A, Bongard F, Putnam B (2012) Arginine vasopressin significantly increases the rate of successful organ procurement in potential donors. Am J Surg 204(6):856-860

21. Macdonald PS, Aneman A, Bhonagiri D, Jones D, O'Callaghan G, Silvester W, Watson A, Dobb G (2012) A systematic review and meta-analysis of clinical trials of thyroid hormone administration to brain dead potential organ donors. Crit Care Med 40(5):1635-1644

22. Novitzky D, Mi Z, Sun Q, Collins JF, Cooper DKC (2014) Thyroid hormone therapy in the management of 63,593 brain-dead organ donors: a retrospective analysis. Transplantation 98(10):1119-1127

23. Rosendale JD, Kauffman HM, McBride MA et al (2003) Aggressive pharmacologic donor management results in more transplanted organs. Transplantation 75(4):482-487

24. Pearson A, Robertson-Malt S, Walsh K, Fitzgerald M (2001) Intensive care nurses' experiences of caring for brain dead organ donor patients. J Clin Nurs 10(1):132-139 